\title{
African Swine Fever Virus Multigene Family Genes Inhibit the Type-I Interferon Response by Acting on the NFאB and IRF3 Signalling Pathways at the Transcription Factor Level or below ${ }^{\dagger}$
}

\author{
Samuel Connell 1,2,*, Ana Reis ${ }^{2}$, Anusyah Rathakrishnan ${ }^{2}$, Sarah Gilbert ${ }^{1}$ and Linda Dixon ${ }^{2}$ \\ 1 Nuffield Department of Medicine, Jenner Institute, University of Oxford, Oxford OX3 7DQ, UK; \\ samuel.connell@pirbright.ac.uk (S.C.); sarah.gilbert@ndm.ox.ac.uk (S.G.) \\ 2 The Pirbright Institute, Pirbright GU24 0NF, UK; anusyah.rathakrishnan@pirbright.ac.uk (A.R.); \\ ana.reis@pirbiright.ac.uk (A.R.); Linda.Dixon@pirbright.ac.uk (L.D.) \\ * Correspondence: samuel.connell@pirbright.ac.uk \\ † Presented at Viruses 2020-Novel Concepts in Virology, Barcelona, Spain, 5-7 February 2020.
}

Published: 15 June 2020

Keywords: African Swine Fever Virus; innate immunity; Viral Host Modulation

African Swine Fever Virus (ASFV) is a haemorrhagic infection of swine, which routinely displays $100 \%$ lethality. ASFV is rapidly spreading through East Asia and the EU, posing an existential threat to the global pork industry. No vaccine presently exists.

ASFV is a large dsDNA virus with a $190 \mathrm{~kb}$ genome, encoding for over 160 open reading frames. The terminal genome ends encode numerous paralogous genes known as multigene family (MGF) genes, which are divided into five clusters $(100,110,300,360,505)$. The composition of the MGFs present varies largely between isolates and are thought to be responsible for isolate virulence. Previous research has unveiled a role in the modulation of the innate immune system, although the functional mechanism of which remains elusive. Inoculation with naturally attenuated OURT88/3 and the genetically modified attenuated Benin $\triangle$ MGF, both lacking six MGF360 and four MGF505 genes, induces protection upon challenge from the parental virus, but has also been demonstrated to induce interferon (IFN) production. Furthermore, increased sensitivity of viral replication to pretreatment of cells with type-I IFN is also observed. This previous work has hinted towards the MGF genes' ability to subvert the interferon pathway.

We aim to elucidate the functional mechanism of the MGF genes responsible for attenuation, in order to rationally generate a live attenuated vaccine. We have demonstrated that the MGF genes of interest display strong inhibition of both the NFKB and IRF3 signalling pathways, seemingly by acting at the transcription factor level or below. Furthermore, the interaction of MGFs with host proteins, and the impact on host IFN responses are under investigation.

(C) 2020 by the authors. Licensee MDPI, Basel, Switzerland. This article is an open access article distributed under the terms and conditions of the Creative Commons Attribution (CC BY) license (http://creativecommons.org/licenses/by/4.0/). 